\title{
Appendices
}

\section{Atmospheric concentrations and sources of PCBs, PCNs, and PCDD/Fs near ranches on a steppe in North China}

Xinrui Yang ${ }^{\mathrm{a}}$, Jicheng $\mathrm{Hu}^{\mathrm{a}, \mathrm{b}, *}$, Wenming Chen ${ }^{\mathrm{a}}$, Te $\mathrm{Bu}^{\mathrm{a}}$, Jun Jin ${ }^{\mathrm{a}, \mathrm{b}}$

${ }^{a}$ College of Life and Environmental Science, MinZu University of China, Beijing 100081, China

${ }^{\mathrm{b}}$ Key Laboratory of Ecology and Environment in Minority Areas (Minzu University of China), National Ethnic Affairs Commission, Beijing 100081, China

* Corresponding author

E-mail: jichhu@126.com 
Table S1 Sampling information of atmospheric samples from family ranches and other points.

Table S2 Internal standard information of PCBs, PCNs, and PCDD/Fs.

Table S3 Atmospheric concentrations of PCBs, PCNs and PCDD/Fs in family ranches and other points.

Figure S1. Concentrations and contributions of PCB (a), PCN (b) and PCDD/F (c, d) homologues.

Figure S2. PCB congener profiles in Factor 1 and from the burning of wood (a); ${ }^{1}$ PCB congener profiles in Factor 2 and in the Aroclors1242 (b). ${ }^{2} *$ refer to this congener are not reported in Lee et al. of emission factors of PCBs from the burning of wood.

Text S1 Positive matrix factorization model

Figure S3. Input data statistics obtained from PMF model of PCBs.

Figure S4. Factor profiles obtained from PMF model of PCBs.

Figure S5. Input data statistics obtained from PMF model of PCNs.

Figure S6. Factor profiles obtained from PMF model of PCNs. 


\section{Table S1}

Sampling information of atmospheric samples from family ranches and other points.

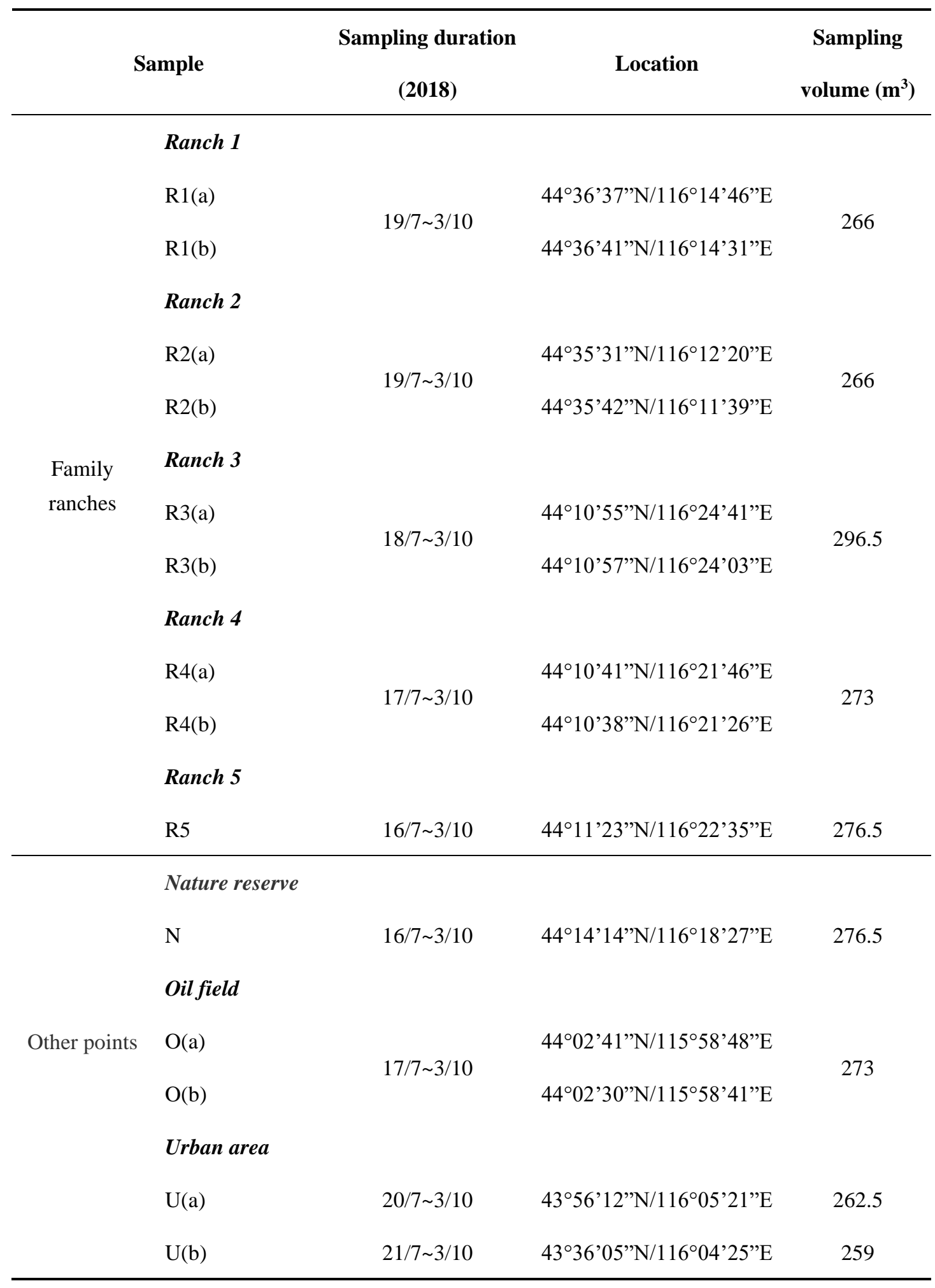




\section{Table S2}

Internal standard information of PCBs, PCNs, and PCDD/Fs.

\begin{tabular}{|c|c|c|c|}
\hline \multicolumn{3}{|c|}{ PCBs CLEAN-UP STANDARD (CE-5045, $\left.{ }^{13} \mathrm{C}_{12}\right)$} & \multirow[b]{2}{*}{ PCB-81 } \\
\hline PCB-28 & PCB-52 & PCB-77 & \\
\hline PCB-101 & PCB-105 & PCB-114 & PCB-118 \\
\hline PCB-123 & PCB-126 & PCB-156 & PCB-157 \\
\hline PCB-167 & PCB-169 & PCB-170 & PCB-180 \\
\hline \multicolumn{4}{|l|}{ PCB-189 } \\
\hline \multicolumn{4}{|c|}{ TETRA-OCTA PCN MIXTURE (ECN-5102, ${ }^{13} \mathrm{C}_{10}$ ) } \\
\hline $1,2,3,4-$ TetraCN & $1,3,5,7-$ TetraCN & $1,2,3,5,7-\mathrm{PentaCN}$ & $1,2,3,5,6,7-\mathrm{HexaCN}$ \\
\hline $1,2,3,4,5,6,7-\mathrm{HeptaCN}$ & OctaCN & & \\
\hline \multicolumn{4}{|c|}{ PCDD/Fs STANDARD (DF-LCS-C, ${ }^{13} \mathrm{C}_{12}$ ) } \\
\hline 2,3,7,8-TCDF & $2,3,7,8-\mathrm{TCDD}$ & $1,2,3,7,8-\mathrm{PeCDF}$ & $2,3,4,7,8-\mathrm{PeCDF}$ \\
\hline $1,2,3,7,8-\mathrm{PeCDD}$ & $1,2,3,4,7,8-\mathrm{HxCDF}$ & $1,2,3,6,7,8-\mathrm{HxCDF}$ & 2,3,4,6,7,8-HxCDF \\
\hline $1,2,3,7,8,9-\mathrm{HxCDF}$ & $1,2,3,4,7,8-\mathrm{HxCDD}$ & $1,2,3,6,7,8-\mathrm{HxCDD}$ & $1,2,3,7,8,9-\mathrm{HxCDD}$ \\
\hline $1,2,3,4,6,7,8-\mathrm{HpCDF}$ & $1,2,3,4,7,8,9-\mathrm{HpCDF}$ & $1,2,3,4,6,7,8-\mathrm{HpCDD}$ & OCDF \\
\hline OCDD & & & \\
\hline
\end{tabular}




\section{Table S3}

Atmospheric concentrations of PCBs, PCNs and PCDD/Fs of family ranches and other points.

\begin{tabular}{|c|c|c|c|c|}
\hline \multirow{2}{*}{\multicolumn{2}{|c|}{ Sample }} & \multicolumn{3}{|c|}{$\Sigma$ Congener $\left(\mathrm{fg} / \mathrm{m}^{3}\right)$} \\
\hline & & \multirow[t]{2}{*}{ PCBs } & \multirow[t]{2}{*}{ PCNs } & \multirow[t]{2}{*}{ PCDD/Fs } \\
\hline & Ranch & & & \\
\hline & $\mathrm{R} 1(\mathrm{a})$ & 1615 & 725 & 5.47 \\
\hline & $\mathrm{R} 1(\mathrm{~b})$ & 1381 & 882 & 12.1 \\
\hline & Mean & 1498 & 803 & 8.76 \\
\hline & Ranch & & & \\
\hline & $\mathrm{R} 2(\mathrm{a})$ & 2030 & 637 & 13.1 \\
\hline & $\mathrm{R} 2(\mathrm{~b})$ & 1454 & 639 & 15.7 \\
\hline & Mean & 1742 & 638 & 14.3 \\
\hline \multirow[t]{13}{*}{ Family ranches } & Ranch & & & \\
\hline & $\mathrm{R} 3(\mathrm{a})$ & 2212 & 774 & 16.6 \\
\hline & $\mathrm{R} 3(\mathrm{~b})$ & 2124 & 703 & 14.6 \\
\hline & Mean & 2168 & 739 & 15.6 \\
\hline & Ranch & & & \\
\hline & $\mathrm{R} 4(\mathrm{a})$ & 2181 & 366 & 19.2 \\
\hline & $\mathrm{R} 4(\mathrm{~b})$ & 1424 & 342 & 17.3 \\
\hline & Mean & 1802 & 454 & 18.2 \\
\hline & Ranch & & & \\
\hline & $\mathrm{R} 5$ & 1498 & 553 & 15.3 \\
\hline & Nature & & & \\
\hline & $\mathrm{N}$ & 1595 & 492 & 14.1 \\
\hline & Oil fiel & & & \\
\hline \multirow[t]{4}{*}{ Other points } & $\mathrm{O}(\mathrm{a})$ & 2156 & 1381 & 22.1 \\
\hline & $\mathrm{O}(\mathrm{b})$ & 1904 & 894 & 14.1 \\
\hline & Mean & 2030 & 1137 & 18.1 \\
\hline & Urban & & & \\
\hline
\end{tabular}




$\begin{array}{llll}\mathrm{U}(\mathrm{a}) & 3542 & 4592 & 46.3 \\ \mathrm{U}(\mathrm{b}) & 9552 & 7275 & 48.6 \\ \text { Mean } & 6547 & 5934 & 47.4\end{array}$



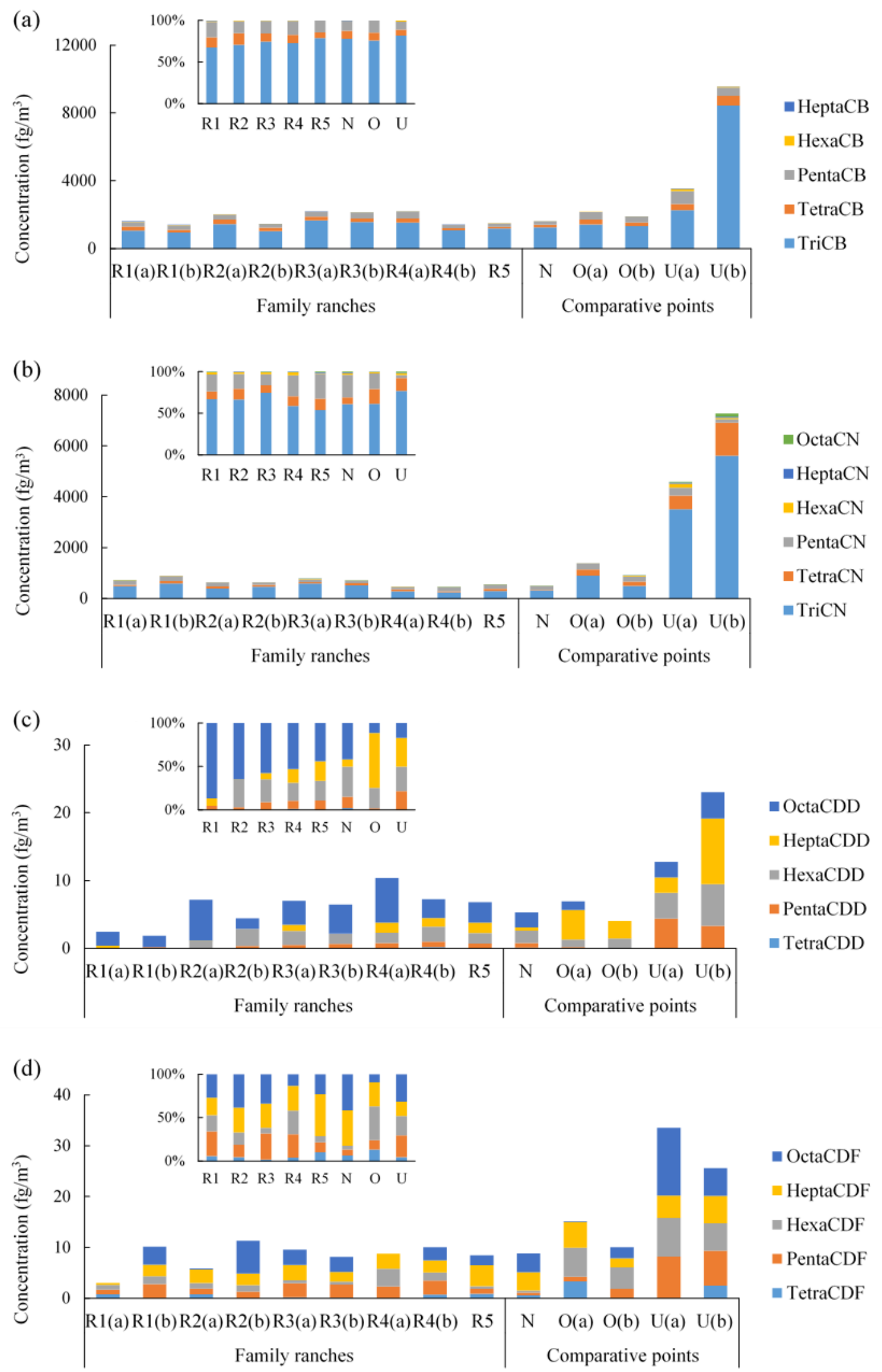

Figure S1. Concentrations and contributions of PCB (a), PCN (b) and PCDD/F (c, d) homologues. 

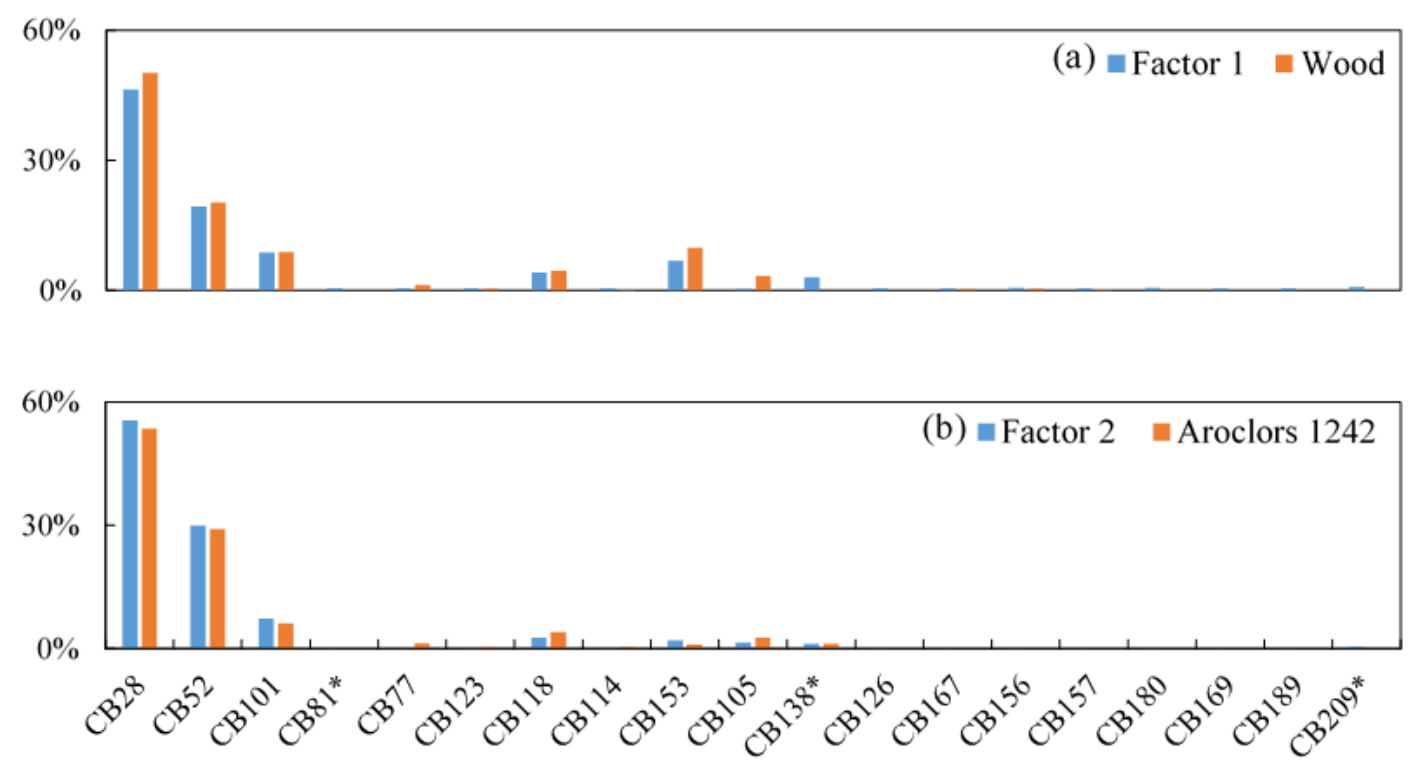

Figure S2. PCB congener profiles in Factor 1 and from the burning of wood (a) ${ }^{1}$ PCB congener profiles in Factor 2 and in the Aroclors1242 (b). ${ }^{2} *$ refer to this congener are not reported in Lee et al. of emission factors of PCBs from the burning of wood.

\section{Text S1 Positive matrix factorization model}

Positive matrix factorization model (PMF) was developed by the United States Environmental Protection Agency, and it can be used to identify the possible sources of POPs. ${ }^{3,4}$ Two files need to be put into PMF: (1) the concentration of each congener in samples and (2) the initial uncertainties of each congener in samples. For congeners whose concentration was lower than or equal to the detection limit, replaced their concentrations with one-half of the method detection limit (MDL) because negative values and zero are not permitted in the input files of PMF., ${ }^{5,6}$ The initial uncertainties (Unc) of them was calculated using a fixed fraction of the MDL (Equation 1) $)^{6}$ :

$$
\mathrm{Unc}=\frac{5}{6} \times M D L
$$

For congeners whose concentration was greater than the MDL, the initial uncertainties were based on the following equation and the Error fraction was set as $10 \%$ $4,5,7$ : 


$$
\mathrm{Unc}=\sqrt{(\text { Error Fraction } \times \text { concentrarion })^{2}+(0.5 \times M D L)^{2}}
$$

Initial uncertainty of each congener would be revised according to the $\mathrm{S} / \mathrm{N}$ ratio before PMF run, which indicates whether the variability in MPF calculation within the noise or not. ${ }^{6}$ Categorized the conger as "Weak" if $\mathrm{S} / \mathrm{N} \leq 1$, and the uncertainty for that congener categorized "Weak" updated to three times the original uncertainty.

Q (robust) and Q (true) values are two important parameter obtained after PMF run. The Q (true) is the goodness-of-fit parameter calculated including all points, and the Q (robust) is the goodness-of-fit parameter calculated excluding points not fit by the model. ${ }^{6}$ Saba et al. ${ }^{5}$ have reported that when Q (true) values were less than 1.5 times the Q (robust) values, the model results can be regarded as not affected by outliers. Besides, EPA PMF 5.0 User Guide have stated that the lowest Q (robust) value represents the most optimal solution from the multiple runs, because it is not affected by points not fit by the model. This means that the lowest Q (robust) value can be a critical parameter for choosing optimal number of factors.

The PMF simulations of PCBs and PCNs both got the lowest Q (robust) at 3 factors. The simulation was conducted using 20 iterations and a random seed generator (default PMF parameters) and the $\mathrm{Q}$ (true) value was less than 1.5 times the $\mathrm{Q}$ (robust) value. Some of the outputs from the PMF are showed below. 


\begin{tabular}{|c|c|c|c|c|c|c|c|c|c|c|}
\hline Species & Cat & $\mathrm{S} / \mathrm{N}$ & Min & 25 th & 50th & 75 th & $\operatorname{Max}$ & $\begin{array}{l}\text { \%odeled } \\
\text { Samples }\end{array}$ & $\begin{array}{l}\text { \% Raw } \\
\text { Samples }\end{array}$ & \\
\hline CB-28 & Strong & 9.0 & 672.48120 & 704.96241 & 825.18682 & 1109.97574 & 1193.32096 & $100.00 \%$ & $100.00 \%$ & \\
\hline CB-52 & Strong & 9.0 & 276.42613 & 325.69235 & 356.74127 & 439.77356 & 469.01670 & $100.00 \%$ & $100.00 \%$ & \\
\hline CB-101 & Strong & 8.9 & 103.96374 & 138.52433 & 211.19813 & 229.63703 & 259.34967 & $100.00 \%$ & $100.00 \%$ & \\
\hline CB-81 & Weak & 0.0 & 1.70000 & 1.70000 & 1.70000 & 1.70000 & 2.37477 & $100.00 \%$ & $100.00 \%$ & \\
\hline CB- 77 & Weak & 0.2 & 1.70000 & 1.70000 & 1.70000 & 2.07644 & 4.85890 & $100.00 \%$ & $100.00 \%$ & \\
\hline CB-123 & Weak & 0.7 & 1.70000 & 1.70000 & 2.40602 & 4.67439 & 4.82765 & $100.00 \%$ & $100.00 \%$ & \\
\hline CB-118 & Strong & 8.6 & 29.84993 & 56.35768 & 75.99258 & 94.54545 & $\mid 110.06137$ & $100.00 \%$ & $100.00 \%$ & \\
\hline CB-114 & Strong & 2.3 & 3.51648 & 4.76596 & 5.93692 & 6.63216 & 9.65531 & $100.00 \%$ & $100.00 \%$ & \\
\hline CB-153 & Strong & 8.9 & 88.79501 & 94.15170 & 102.11503 & 118.09003 & 134.50930 & $100.00 \%$ & $100.00 \%$ & \\
\hline CB-105 & Strong & 5.5 & 1.70000 & 8.24559 & 22.56030 & 31.53331 & 39.83986 & $100.00 \%$ & $100.00 \%$ & \\
\hline CB-138 & Strong & 8.5 & 28.05575 & 42.42703 & 63.88439 & 89.50288 & 110.42672 & $100.00 \%$ & $100.00 \%$ & \\
\hline CB-126 & Weak & 0.0 & 1.14172 & 1.70000 & 1.70000 & 1.70000 & 1.99907 & $100.00 \%$ & $100.00 \%$ & \\
\hline CB-167 & Weak & 0.0 & 1.70000 & 1.70000 & 1.70000 & 1.70000 & 1.70000 & $100.00 \%$ & $100.00 \%$ & \\
\hline CB-156 & Weak & 0.8 & 1.70000 & 1.70000 & 1.70000 & 3.56215 & 10.85831 & $100.00 \%$ & $100.00 \%$ & \\
\hline CB-157 & Weak & 0.1 & 1.70000 & 1.70000 & 1.70000 & 2.06082 & 3.62465 & $100.00 \%$ & $100.00 \%$ & \\
\hline CB-180 & Strong & 3.1 & 1.70000 & 3.63246 & 5.60626 & 14.26764 & 17.81076 & $100.00 \%$ & $100.00 \%$ & \\
\hline CB-169 & Weak & 0.0 & 1.70000 & 1.70000 & 1.70000 & 1.70000 & 1.70000 & $100.00 \%$ & $100.00 \%$ & \\
\hline CB-189 & Weak & 0.5 & 1.70000 & 1.70000 & 1.70000 & 2.63108 & 8.43668 & $100.00 \%$ & $100.00 \%$ & \\
\hline CB-209 & Strong & 2.7 & 1.70000 & 1.70000 & 4.74954 & 17.10290 & 25.26316 & $100.00 \%$ & $100.00 \%$ & \\
\hline Unsort & \multicolumn{3}{|c|}{ Species Category Settings: } & Strong & Weak & Bad & \multicolumn{2}{|c|}{ Total Variable (Defaults to Weak) } & & 0 \\
\hline Help | & \multicolumn{3}{|c|}{ Strong Species: 10} & & \multicolumn{3}{|c|}{ Weak Species: 9} & \multicolumn{2}{|c|}{ Bad Species: 0} & \\
\hline
\end{tabular}

Figure S3. Input data statistics obtained from PMF model of PCBs.

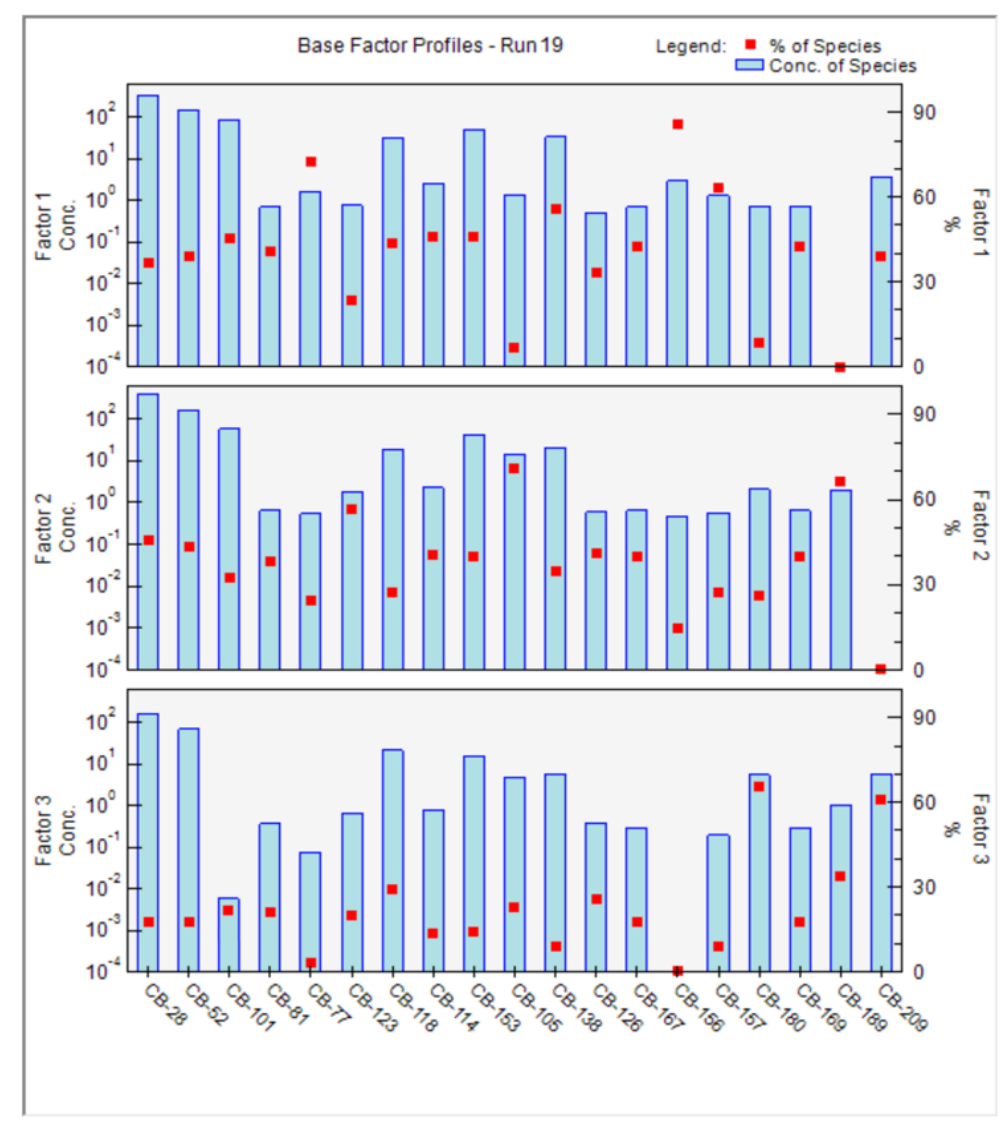

Figure S4. Factor profiles obtained from PMF model of PCBs. 


\begin{tabular}{|c|c|c|c|c|c|c|c|c|c|}
\hline \multicolumn{10}{|c|}{ Input Data Statistios } \\
\hline Species & Cat & $S / N$ & Mn & $25 \mathrm{~h}$ & $50 \mathrm{~h}$ & $79 \mathrm{~h}$ & Max & $\begin{array}{l}\text { YModeled } \\
\text { Samples }\end{array}$ & $\begin{array}{l}\text { YRaw } \\
\text { Samples }\end{array}$ \\
\hline $\mathrm{CN}_{20}$ & Strong & 9.8 & 16.63653 & 23.00328 & 41.70735 & 58.16912 & 62.33766 & $100.00 \%$ & $100.00 \%$ \\
\hline CN19 & Strong & 26 & 0.92500 & 225229 & 4.74075 & 10.26633 & 15.02783 & $100.00 \%$ & $100.00 \%$ \\
\hline CN21 & Weak & 0.8 & 0.92500 & 1.37825 & 2.96846 & 3.96283 & 8.16327 & $100.00 \%$ & $100.00 \%$ \\
\hline CN24/14 & Strong & 10.0 & 52.98749 & 87.88455 & 203.34196 & 27277488 & 343.78479 & $10000 \%$ & $100.00 \%$ \\
\hline CN15 & Strong & 3.2 & 0.92500 & 0.92500 & 0.92500 & 17.43970 & 30.22166 & $100.00 \%$ & $100.00 \%$ \\
\hline CN16 & Srong & 5.1 & 0.92500 & 4.99097 & 9.40325 & 18.99473 & 35.62152 & $100.00 \%$ & $100.00 \%$ \\
\hline $\mathrm{CN}_{17 / 25 / 26}$ & Srong & 8.5 & 7.40471 & 14.55568 & 23.00557 & 43.36613 & 63.50942 & $100.00 \%$ & $100.00 \%$ \\
\hline $\mathrm{CN} 13$ & Srong & 10.0 & 2804537 & 43.96437 & 71.98516 & 109.27644 & 125.61029 & $10000 \%$ & $100.00 \%$ \\
\hline CN22 & Weak & 0.6 & 0.92500 & 0.92500 & 1.79182 & 297619 & 8.86076 & $100.00 \%$ & $100.00 \%$ \\
\hline $\mathrm{CN}_{23}$ & Srong & 6.4 & 0.92500 & 5.68904 & 16.41577 & 27.27151 & 39.14901 & $100.00 \%$ & $100.00 \%$ \\
\hline CN18 & Weak & 0.5 & 0.92500 & 1.48207 & 1.85529 & 3.49648 & 5.82707 & $10000 \%$ & $100.00 \%$ \\
\hline $\mathrm{CN} 42$ & Weak & 0.0 & 0.92500 & 0.92500 & 0.92500 & 0.92500 & 0.92500 & $100.00 \%$ & $100.00 \%$ \\
\hline CN37/33/34 & Srong & 6.4 & 3.57143 & 7.71641 & 15.03759 & 23.16174 & 27.64378 & $100.00 \%$ & $100.00 \%$ \\
\hline $\mathrm{CN}_{44 / 47}$ & Strong & 5.0 & 5.44693 & 6.64486 & 10.56049 & 14.96031 & 21.70686 & $100.00 \%$ & $100.00 \%$ \\
\hline CN45/36 & Strong & 25 & 0.92500 & 1.53305 & 5.00928 & 9.90138 & 17.80841 & $100.00 \%$ & $100.00 \%$ \\
\hline $\mathrm{CN} 28 / 43$ & Weak & 0.1 & 0.78361 & 0.92500 & 0.92500 & 0.92500 & 3.60316 & $100.00 \%$ & $100.00 \%$ \\
\hline $\mathrm{CN}_{29}$ & Srong & 6.1 & 0.92500 & 681407 & 15.02783 & \begin{tabular}{|l|l|} 
& 19.38287
\end{tabular} & 29.33796 & $100.00 \%$ & $100.00 \%$ \\
\hline CN27 & Weak & 0.3 & 4.62500 & 4.62500 & 5.56111 & 17.14188 & 19.58549 & $100.00 \%$ & $100.00 \%$ \\
\hline CN39 & Srong & 1.7 & 0.92500 & 0.92500 & 2.16059 & 9.14828 & 1300166 & $100.00 \%$ & $100.00 \%$ \\
\hline CN32 & Weak & 0.0 & 0.92500 & 0.92500 & 0.92500 & 0.93975 & 1.33044 & $10000 \%$ & $100.00 \%$ \\
\hline $\mathrm{CN} 48 / 35$ & Weak & 0.3 & 0.92500 & 0.92500 & 1.69173 & 288912 & 4.33996 & $100.00 \%$ & $100.00 \%$ \\
\hline CN38/40 & Weak & 0.7 & 0.92500 & 0.92500 & 1.29870 & 3.45425 & 9.99902 & $100.00 \%$ & $100.00 \%$ \\
\hline N4E & tron & 19 & 9252500 & 1.9995 & 5 & 89044 & 9 & 00.00 & 100 \\
\hline
\end{tabular}

\begin{tabular}{|c|c|c|c|c|c|c|c|c|c|}
\hline CN31 & Weak & 0.4 & 0.92500 & 0.92500 & 0.92500 & 257299 & 7.04033 & $100.00 \%$ & $100.00 \%$ \\
\hline $\mathrm{CN} 41$ & Weak & 0.0 & 0.92500 & 0.92500 & 0.92500 & 0.92500 & 0.92500 & $100.00 \%$ & $100.00 \%$ \\
\hline CN52 & Weak & 0.7 & 3.70000 & 3.70000 & 4.26716 & 8.72855 & 50.81374 & $100.00 \%$ & $100.00 \%$ \\
\hline CN58 & Strong & 4.5 & 0.92500 & 1.90401 & 7.60668 & 22.16358 & 24.77396 & $100.00 \%$ & $100.00 \%$ \\
\hline CN61 & Strong & 5.6 & 0.92500 & 0.92500 & 14.83742 & 83.08953 & 105.70013 & $100.00 \%$ & $100.00 \%$ \\
\hline CN50 & Strong & 5.5 & 0.92500 & 5.62445 & 13.35807 & 17.65953 & 20.48872 & $100.00 \%$ & $100.00 \%$ \\
\hline CN51 & Strong & 3.1 & 0.92500 & 0.92500 & 6.01504 & 14.48833 & 22.96564 & $100.00 \%$ & $10000 \%$ \\
\hline CN54 & Weak & 0.0 & 0.92500 & 0.92500 & 0.92500 & 0.92500 & 204082 & $100.00 \%$ & $10000 \%$ \\
\hline CN57 & Strong & 30 & 0.92500 & 1.87227 & 8.79121 & 10.77853 & 11.87384 & $100.00 \%$ & $100.00 \%$ \\
\hline CN62 & Strong & 3.3 & 0.92500 & 0.92500 & 6.30798 & 14.71780 & 29.76760 & $100.00 \%$ & $100.00 \%$ \\
\hline CN53/55 & Strong & 22 & 0.92500 & 0.92500 & 0.92500 & 10.71429 & 26.45076 & $100.00 \%$ & $100.00 \%$ \\
\hline CN59 & Strong & 24 & 0.00000 & 0.92500 & 1.31579 & 11.46404 & 17.33717 & $100.00 \%$ & $100.00 \%$ \\
\hline CN49 & Strong & 20 & 0.92500 & 0.92500 & 0.92500 & 7.42481 & 22.36842 & $100.00 \%$ & $100.00 \%$ \\
\hline CN56 & Strong & 5.4 & 0.92500 & 0.92500 & 14.46886 & 43.55166 & 63.38248 & $100.00 \%$ & $100.00 \%$ \\
\hline CN66/67 & Weak & 0.8 & 0.22780 & 0.65047 & 0.92500 & 6.20301 & 6.98349 & $100.00 \%$ & $100.00 \%$ \\
\hline CN64/68 & Strong & 2.3 & 0.92500 & 0.92500 & 2.92802 & 10.70358 & 15.22556 & $100.00 \%$ & $100.00 \%$ \\
\hline CN69 & Weak & 0.4 & 0.92500 & 0.92500 & 0.92500 & 256767 & 6.30798 & $100.00 \%$ & $100.00 \%$ \\
\hline CN71/72 & Weak & 0.9 & 0.90862 & 0.92500 & 226052 & 4.54630 & 9.59379 & $100.00 \%$ & $100.00 \%$ \\
\hline CN63 & Weak & 0.1 & 0.92500 & 0.92500 & 0.92500 & 0.93242 & 3.19549 & $100.00 \%$ & $100.00 \%$ \\
\hline CN65/70 & Weak & 0.9 & 0.92500 & 0.92500 & 0.92500 & 4.32663 & 12.21805 & $100.00 \%$ & $100.00 \%$ \\
\hline CN73 & Weak & 0.8 & 0.91575 & 1.96392 & 3.38346 & 4.51604 & 4.57875 & $100.00 \%$ & $100.00 \%$ \\
\hline CN74 & Weak & 0.0 & 0.92500 & 0.92500 & 0.92500 & 0.92500 & 0.93985 & $100.00 \%$ & $100.00 \%$ \\
\hline CN75 & Weak & 0.0 & 4.26716 & 4.62500 & 4.62500 & 4.62500 & 7.70677 & $100.00 \%$ & $100.00 \%$ \\
\hline
\end{tabular}

Figure S5. Input data statistics obtained from PMF model of PCNs.

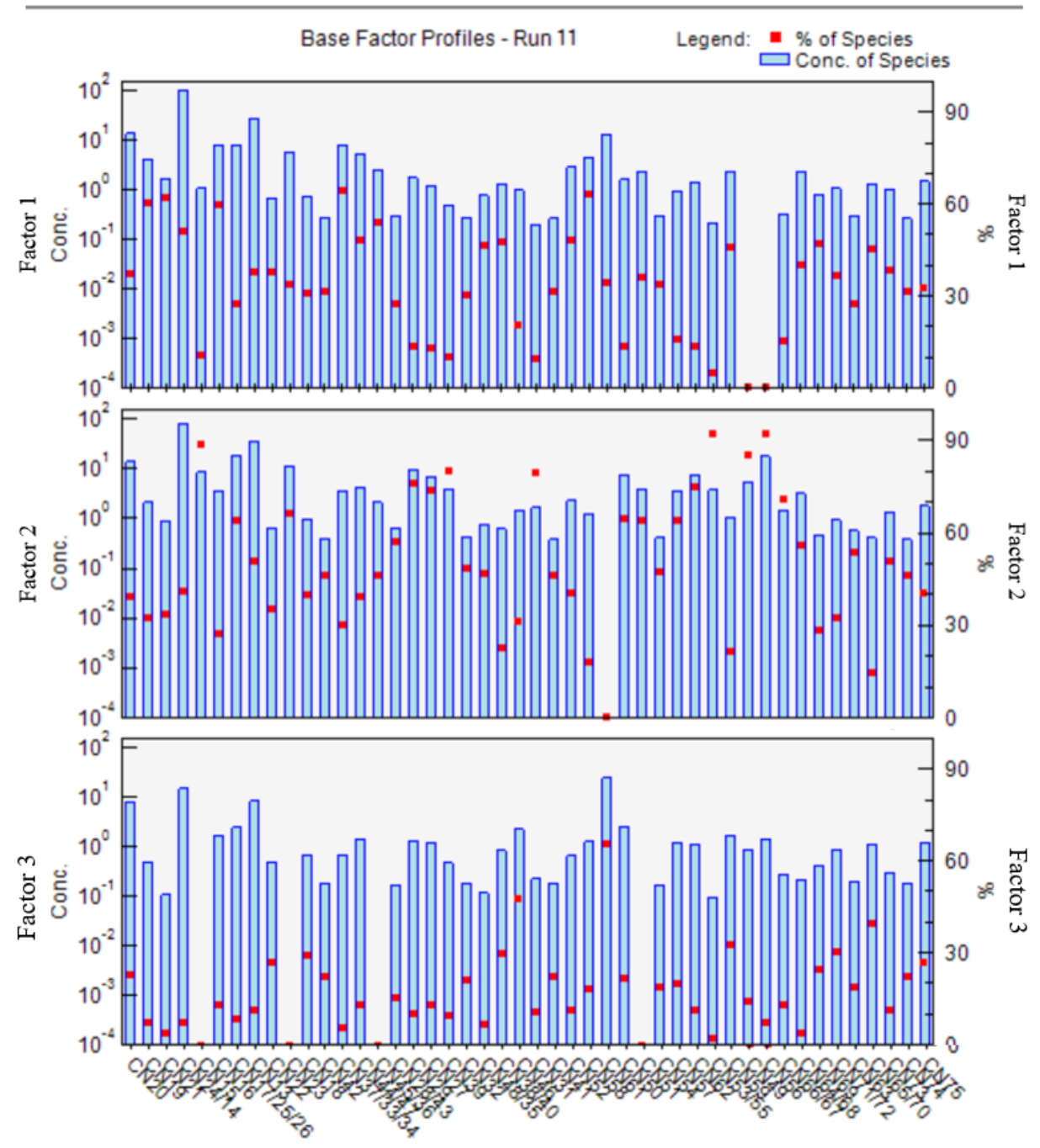

Figure S6. Factor profiles obtained from PMF model of PCNs. 


\section{Reference:}

(1) Lee, R.G.M., Coleman, P., Jones, J.L., Jones, K.C., Lohmann, R., 2005. Emission factors and importance of $\mathrm{PCDD} / \mathrm{Fs}, \mathrm{PCBs}, \mathrm{PCNs}$, PAHs and $\mathrm{PM}_{10}$ from the domestic burning of coal and wood in the U.K. Environmental Science \& Technology 39, 1436-1447.

(2) Frame, G.M., Cochran, J.W., Boewadt, S.S., 1996. Complete PCB congener distributions for 17 Aroclor mixtures determined by 3 HRGC systems optimized for comprehensive, quantitative, congener-specific analysis. Journal of High Resolution Chromatography 19(12), 657-668.

(3) Paatero, P., Tapper, U., 1994. Positive matrix factorization: A non-negative factor model with optimal utilization of error estimates of data values. Environmetrics, 5(2), 111-126.

(4) Cetin, B., Yurdakul, S., Gungormus, E., Ozturk, F., Sofuoglu, S.C., 2018. Source apportionment and carcinogenic risk assessment of passive air sampler-derived PAHs and PCBs in a heavily industrialized region. Science of the Total Environment 633, 30-41.

(5) Saba, T., Su, S., 2013. Tracking polychlorinated biphenyls (PCBs) congener patterns in Newark Bay surface sediment using principal component analysis (PCA) and positive matrix factorization (PMF). Journal of Hazardous Materials 260, 634-643.

(6) US EPA, 2014. EPA Positive Matrix Factorization (PMF) 5.0 Fundamentals and User Guide. EPA/600/R-14/108, EPA, Washington, DC.

(7) US EPA, 2008. EPA Positive Matrix Factorization (PMF) 3.0 Fundamentals \& User Guide. EPA 600/R-08/108, EPA, Washington, DC. 